\title{
Methodology Report Efficient siRNA Delivery by the Cationic Liposome DOTAP in Human Hematopoietic Stem Cells Differentiating into Dendritic Cells
}

\author{
Sabata Martino, Ilaria di Girolamo, Roberto Tiribuzi, Francesco D’Angelo, Alessandro Datti, \\ and Aldo Orlacchio
}

Dipartimento di Medicina Sperimentale e Scienze Biochimiche, Sezione di Biochimica e Biologia Molecolare, Università di Perugia, Via del Giochetto, 06100 Perugia, Italy

Correspondence should be addressed to Aldo Orlacchio, orly@unipg.it

Received 11 December 2008; Accepted 20 March 2009

Recommended by Bibekanand Mallick

RNA interference technology is an ideal strategy to elucidate the mechanisms associated with human CD34 $4^{+}$hematopoietic stem cell differentiation into dendritic cells. Simple manipulations in vitro can unequivocally yield alloreactive or tolerogenic populations, suggesting key implications of biochemical players that might emerge as therapeutic targets for cancer or graft-versushost disease. To knockdown proteins typically involved in the biology of dendritic cells, we employed an siRNA delivery system based on the cationic liposome DOTAP as the carrier. Freshly-isolated CD34 $4^{+}$cells were transfected with siRNA for cathepsin $\mathrm{S}$ with negligible cytotoxicity and transfection rates $(>60 \%)$ comparable to the efficiency shown by lentiviral vectors. Further, cathepsin S knockdown was performed during both cell commitment and through the entire 14-day differentiation process with repeated transfection rounds that had no effect per se on cell development. Tested in parallel, other commercially-available chemical reagents failed to meet acceptable standards. In addition to safe and practical handling, a direct advantage of DOTAP over viral-mediated techniques is that transient silencing effects can be dynamically appraised through the recovery of targeted proteins. Thus, our findings identify DOTAP as an excellent reagent for gene silencing in resting and differentiating CD34 ${ }^{+}$cells, suggesting a potential for applications in related preclinical models.

Copyright ( $) 2009$ Sabata Martino et al. This is an open access article distributed under the Creative Commons Attribution License, which permits unrestricted use, distribution, and reproduction in any medium, provided the original work is properly cited.

\section{Introduction}

The discovery of siRNAs and subsequent advances in RNA interference (RNAi) technology has contributed to a major methodological shift in functional genomics [1]. Gene silencing, that can be successfully extended to highthroughput screening formats $[2,3]$, is a procedure that directly accelerates biological and biomedical research through the identification of molecular players involved in development, homeostasis, and disease. In the new millennium, the explosive growth of this field has also raised the prospect of RNAi as a therapeutic to treat a wide range of pathologies, in particular viral infections, cancer, and neurodegenerative disorders [4-7].

Synthetic siRNAs have emerged as a widespread means for gene silencing in mammalian cells [8], notwithstand- ing limitations associated with a nonrenewable supply, temporary silencing effect, and occurrence of off-target activity $[9,10]$. Next-generation synthetic products were recently introduced to ensure the highest silencing power under a minimal occurrence of false positives (e.g., ONTARGETplus [Dharmacon] and Silencer Select validated siRNAs [Ambion]), and provide the opportunity to transfect virtually any cell type in the absence of a carrier (Accell siRNAs [Dharmacon]). Further, shRNA expression vectors coupled to lentiviral transduction systems were developed to obtain unlimited resources, achieve stable integration into the genome in a widest variety of both dividing and nondividing cells, produce sustained transgene expression, and comply with in vivo applications (e.g., MISSION [Sigma-Aldrich], BLOCK-iT [Invitrogen], SMARTvector [Dharmacon], GeneNet [System Biosciences], and Lenti-X 
[Clontech]) [11-13]. Finally, access to mimic and inhibitor miRNA libraries for use in either gain- or loss-of-function analyses, respectively, have enhanced scope and flexibility of studies intended to unravel the involvement of miRNAs (e.g., PremiR [Ambion], miRIDIAN [Dharmacon], and miScript [Qiagen]) in cytoplasmic posttranscriptional gene silencing [14], translational activation [15] and transcriptional control of gene expression in the nucleus [16].

However, the extraordinary benefits of these breakthroughs in RNAi technology may imply significant research investments and special requirements, such as biocontainment facilities dedicated to viral work. Chemical agents, instead, are relatively inexpensive, easy to handle and generally already optimized by the vendor for use with specific cell lines. Nonetheless, chemical transfections in finite cultures or freshly-isolated primary cells usually raise major challenges due to a combination of cytotoxic effects and poor transfection efficiencies. These problems become more obvious in dynamic systems such as stem cells subjected to differentiation.

Lentiviral vectors can successfully transduce hematopoietic stem cells (HSCs) $[12,17]$, but integrate stably in the genome and preclude the recovery of knocked-down proteins. Within nonviral transfection methods for HSCs, electroporation raises major survival issues due to apoptotic cell death [18], while nucleofactor technology was shown to deliver mRNA and plasmid DNA with inconsistent transfection and cell survival rates [19].

Lipofection has typically been considered inefficient for use with HSCs [20]. Nonetheless, based on the large number of products currently available from vendors and the advantages offered by a transient gene knockdown, we screened a variety of agents with different chemical formulations and found that the liposomal version of the monocationic lipid DOTAP is an excellent vector for the treatment of freshly isolated HSCs. Further, we observed that DOTAP is an efficient vehicle for gene knockdown in HSCs induced to differentiate into immunogenic dendritic cells in vitro through a conventional treatment based on Flt3L, GMCSF, IL-4 and TNF- $\alpha$ [21-23].

\section{Materials and Methods}

2.1. Isolation of Human HSCs. Blood samples were collected from healthy volunteers who provided informed consent. Human peripheral blood mononuclear cells were isolated by density gradient centrifugation over Ficoll-Paque PLUS (GE Healthcare), after which $\mathrm{CD} 34^{+}$cells were purified by immunomagnetic selection using the minimagneticactivated cell sorter (MACS) system (Miltenyi Biotec). Mean purity of CD34-enriched cells, determined by flow cytometry using a FACScan (BD Biosciences), was 94.8\%, with a median value of 92 throughout an 86-98.5 range.

2.2. Differentiation of HSCs into Dendritic Cells. To generate immunogenic dendritic cells, CD34-enriched cells were transferred to $25 \mathrm{~cm}^{2}$ flasks at a density of $10^{5}$ cells $/ \mathrm{mL}$ and cultured for 14 days in RPMI-1640 medium (Euroclone) containing 10\% fetal calf serum (FCS) (Euroclone) and a cytokine cocktail composed of human recombinant, Flt3L ( $50 \mathrm{ng} / \mathrm{mL}), \mathrm{GM}-\mathrm{CSF}(50 \mathrm{ng} / \mathrm{mL}), \mathrm{IL}-4(10 \mathrm{ng} / \mathrm{mL})$, and TNF- $\alpha(2.5 \mathrm{ng} / \mathrm{mL})$ (PeproTech EC). Every third day, half of the culture medium was replaced by fresh medium supplemented with the same cocktail. Cells were analyzed at day 3,7 , and 14 to evaluate the expression of specific markers of differentiation [23].

\subsection{Evaluation of Different Reagents for siRNA Transfection} of Untreated CD $34^{+}$Stem Cells. The following, commercially available reagents were tested to determine transfection rates and cytotoxicity effects: TransPass R1 and TransPass R2 (New England Biolabs), Lipofectamine 2000 and Lipofectamine LTX (Invitrogen), siPORT NeoFX and siPORT Amine (Ambion), and DOTAP (Roche). Tests were performed using a 96-well plate format. Freshly prepared CD34 ${ }^{+}$cells were seeded at a density of $2 \times 10^{4} /$ well. Fluorescein-labeled siRNA control (New England Biolabs), in a final concentration of $15 \mathrm{nM}$, was used to monitor the transfection procedures. Fluorescein-labeled siRNA was regularly diluted with RPMI1640 without serum, except for the experiment performed with DOTAP, in which the diluent was HBS (Hepes-buffered saline: $20 \mathrm{mM}$ HEPES, pH 7.4, containing $150 \mathrm{mM} \mathrm{NaCl}$ ).

The transfection reagents were essentially employed as per each vendor's recommendations. TransPass R1 and TransPass R2 $(0.2 \mu \mathrm{L})$ were diluted directly in the fluoresceinlabeled siRNA solution $(10 \mu \mathrm{L})$, incubated 30 minutes at room temperature and then added to the cultures $(90 \mu \mathrm{L})$. Lipofectamine 2000 and Lipofectamine LTX $(0.25 \mu \mathrm{L})$ were first diluted in $12.5 \mu \mathrm{L}$ RPMI-1640 without serum and, after 5 minutes incubation, mixed with $12.5 \mu \mathrm{L}$ of fluoresceinlabeled siRNA. After 30 minutes incubation, the transfection complexes were added to the cells $(75 \mu \mathrm{L})$.

siPORT NeoFX and siPORT Amine were first diluted (0.5 and $0.3 \mu \mathrm{L}$, resp.) in $10 \mu \mathrm{L}$ RPMI-1640 without serum. After 10 minutes at room temperature, each carrier was mixed with $10 \mu \mathrm{L}$ of fluorescein-labeled siRNA. The mixtures $(20 \mu \mathrm{L})$ were incubated 10 minutes, then spotted in the assay wells. Within 5 minutes, cells were dispensed in a volume of $80 \mu \mathrm{L}$.

DOTAP $(1.4 \mu \mathrm{L})$ was diluted in $7 \mu \mathrm{L}$ HBS and then mixed, very slowly, with the same volume of fluoresceinlabeled siRNA. After 30 minutes at room temperature, the mixture was added to cells ( $186 \mu \mathrm{L})$ resuspended in RPMI1640 containing $20 \%$ FCS.

Except for the experiment performed with DOTAP, fresh medium was added to the assay wells 4 hours after each transfection process in order to achieve a final volume and a concentration of FCS of $200 \mu \mathrm{L}$ and $10 \%$, respectively.

In all instances, transfection and cell survival were evaluated 24 hours after the transfection method.

Transfection rates were expressed as the ratio between transfected (fluorescein-labeled) and total number of viable cells measured via DAPI nuclear staining and trypan blue exclusion test. Signals were captured by a Nikon Eclipse TE2000-S fluorescence microscope equipped with a Cell$\mathrm{F}$ video camera (Olympus) and processed via the Cell-F software. 


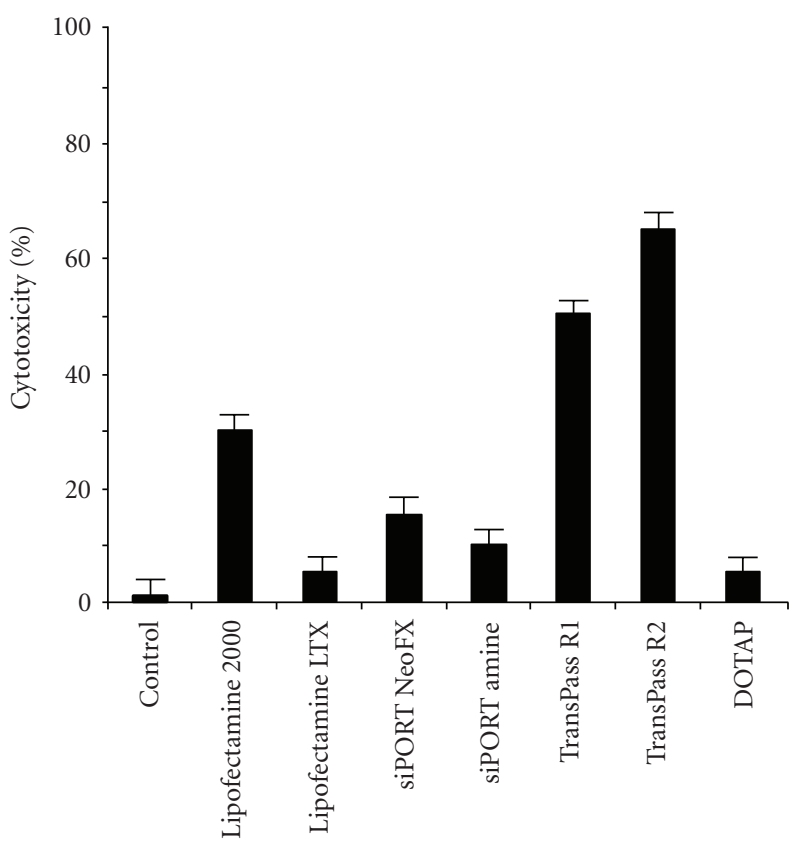

(a)

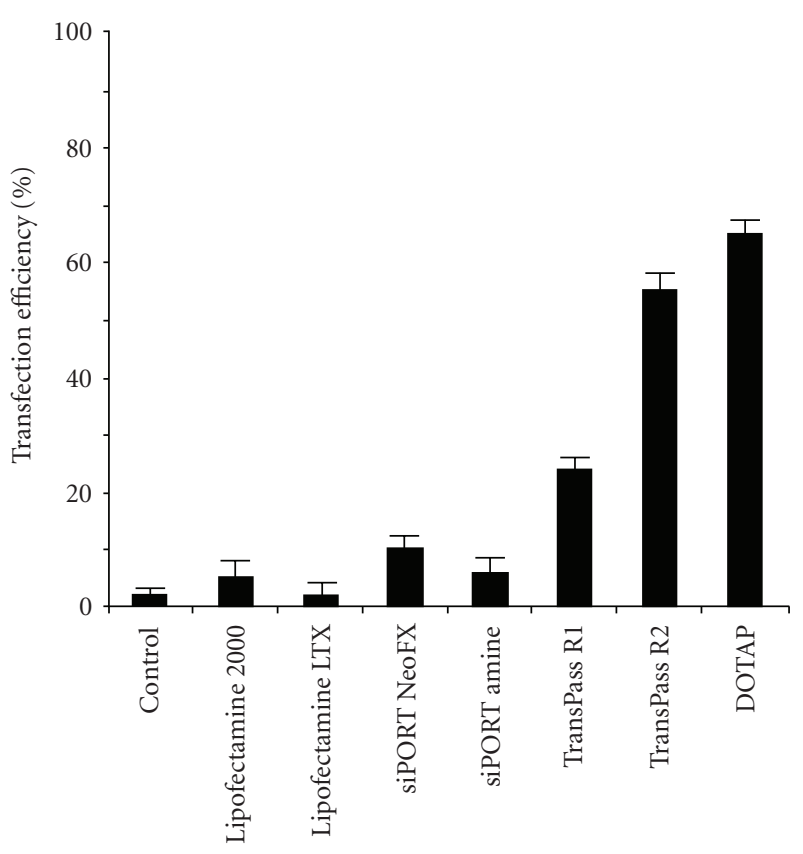

(b)

FIGURE 1: Effects of siRNA delivery agents on cytotoxicity and transfection rates. Freshly isolated HSCs were subjected to agent-specific transfection protocols using a fluorescein-labeled, nontargeting siRNA control as described in the Materials and Methods. After 24 hours incubation, cytotoxicity was assessed through the trypan blue dye exclusion test (a), while transfection rates were calculated from the ratio between fluorescein-labeled and total number of DAPI-stained cells (b). Data shown are representative of triplicate determinations.

2.4. Silencing of Cathepsin S Gene via the DOTAP Reagent in HSCs Subjected to Differentiation. Freshly isolated CD $34^{+}$ cells were resuspended in RPMI-1640 supplemented with the agents used to generate immunogenic dendritic cells. Immediately after treatment, cells were subjected to transfection using a predesigned siRNA targeting the CatS gene (CTSS: ID-113084-113085, Ambion).

Cells subjected to mock- and scrambled-siRNA transfection (ID-46183G, Ambion) under the same experimental conditions were employed for control purposes. The procedure, run in a 24-well plate format via triplicate tests, was implemented using a $25 \mathrm{nM}$ final siRNA concentration. Each well-contained $2 \times 10^{5}$ cells in a volume of $500 \mu \mathrm{L}$. The siRNAs were combined with DOTAP and maintained for 30 minutes at room temperature to form complexes. The mixture $(50 \mu \mathrm{L})$ was then overlaid dropwise on the cell cultures. Following 4-hour incubation, 1.2 mL of RPMI-1640 containing $10 \%$ FCS and differentiating agents was added to each well. To ensure silencing of genes for the entire 14-day differentiation process, cells were centrifuged, resuspended in $500 \mu \mathrm{L}$ of cytokine-enriched culture medium and exposed to a second (day 3) and third (day 9) transfection round under the same experimental conditions.

2.5. Western Blotting. Preparation of cell extracts, electrophoresis (12\% gel) under reducing conditions and Western blotting procedures were performed as previously described [24].

Precursors and mature forms of cathepsin S were analyzed using polyclonal antibodies from Santa Cruz Biotech- nology as the primary antibodies. Immunodetection was carried out by employing the enhanced, chemiluminescent Amersham ECL Plus kit. For each blot, several time exposures were performed to confirm that the results were within the linear response range of the film. Densitometric scans via an MCID system (InterFocus Imaging) were used to demonstrate that the intensity of the bands was proportional to protein content.

\section{Results and Discussion}

Several transfection reagents were employed to evaluate cytotoxic effects and the capacity to deliver a fluoresceinlabeled siRNA control in freshly isolated, untreated HSCs. Commercially-available agents were chosen to explore a variety of structural diversities and chemical formulations as per each vendor's product description. The screen included a cationic lipid (TransPass R1), a nonlipid cationic polymer (TransPass R2), a lipid-based formulation designed for reverse transfection protocols (siPORT NeoFX), a polyamine mixture (siPORT Amine), and different versions of cationic liposomes (Lipofectamine 2000, Lipofectamine LTX), an animal origin-free product, and DOTAP, obtained from N-[1-(2,3-Dioleoyloxy)propyl]N,N,N-trimethylammonium methylsulfate.

Four chemicals (i.e., Lipofectamine LTX, siPORT NeoFX, siPORT Amine, and DOTAP) were relatively well tolerated by HSCs ( $\geq 85 \%$ viability) (Figure $1(\mathrm{a})$ ), however only DOTAP was found to combine a lack of cell cytotoxicity with efficient $(60 \%)$ siRNA transfer (Figure 1(b)). TransPass R2 showed 


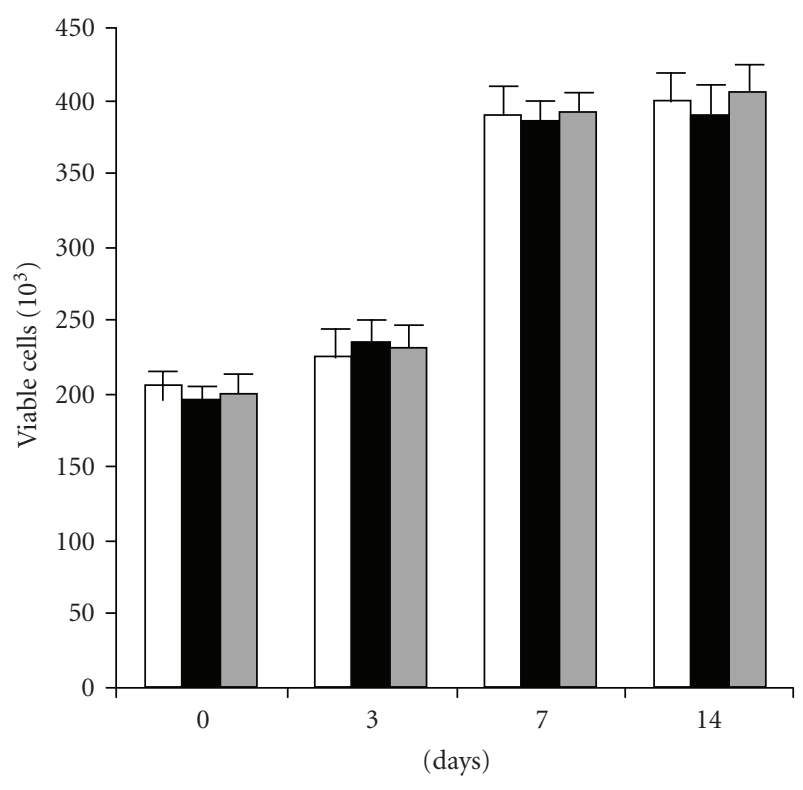

FIGURE 2: Cell viability during repeated, DOTAP-mediated transfection rounds in differentiating HSCs. Freshly isolated HSCs were induced to differentiate into immunogenic dendritic cells as described in the Materials and Methods. Immediately after treatment, cultures were split into two cell populations, of which one, used as the control, was grown in absence of manipulations (white bars), while the other was subjected to two and three DOTAP-based, scrambled siRNA transfection rounds performed at day 0 and day 3 (black bars), and day 0, day 3 and day 9 (grey bars). Cell viability was monitored by trypan blue staining at different time points as indicated.

a transfection capacity similar to DOTAP, but significant cytotoxicity. Thus, DOTAP emerged as the chemical with suitable attributes for use in gene knockdown studies of differentiating HSCs.

We have previously shown that changes in cathepsin $S$ expression correlate with the differentiation of HSCs into immunogenic dendritic cells in vitro. In this regard, cathepsin $\mathrm{S}$ shows a precursor form of $66 \mathrm{KDa}$ in freshly isolated HSCs, while the conventional, mature protein of $26 \mathrm{KDa}$ appears following a 7-day treatment with a cytokine cocktail [22]. Based on the physiological role of cathepsin $S$ in the biology of dendritic cells $[25,26]$, we employed a gene knockdown strategy to investigate the involvement of the enzyme during the entire 14-day differentiation process.

In light of the transient nature of siRNA-mediated silencing, we implemented the DOTAP protocol for three rounds of transfection performed in untreated HSCs (day 0 ) and, following cocktail treatment, in cells subjected to differentiation for 3 and 9 days. Trypan blue exclusion tests performed at different time points showed that multiple knockdowns did not affect cell viability (Figure 2). Furthermore, overall metabolic activity and cell proliferation were also unchanged, measured through reduction rates of the tetrazolium salt XTT (not shown) [27]. We also observed that repeated transfections of a scrambled siRNA had no impact on the temporal expression of antigens typically modulated during the differentiation of HSCs into phenotypically and functionally mature dendritic cells, namely CD34, CD1a, CD80, CD86, CD83, and CD11c (Figure 3). Similarly, the expression of additional immunophenotypic markers such as CD40 (10.04\% \pm 0.5$), C D 4(59.02 \% \pm 0.3), C D 123(46.08 \% \pm$ $0.6)$ HLA-ABC $(94.10 \% \pm 0.7)$ in fully differentiated dendritic cells was found to compare well with previous data [23], suggesting that the differentiation process advanced normally within expected temporal timeframes. Further, mixed lymphocyte reaction tests (not shown) did not reveal any discrepancy in the functional properties of alloreactive dendritic cells [23]. On day 14, the reduction of cathepsin $\mathrm{S}$ expression exceeded $95 \%$ in the differentiation model, as indicated by Western blotting analysis (Figure 4(a)). Instead, gene silencing performed only twice at day 0 and, again, at day 3 caused more than $80 \%$ enzyme reduction during the cell commitment stage (day 7), followed by increasing cathepsin levels that resulted in a fully restored expression after two weeks (Figure 4(b)). Interestingly, no differences on the CD34, CD1a, CD80, CD86, CD83, and CD11c markers were measured during HSCs differentiation subjected to two and three transfection rounds compared to untrasfected cells (Figure 3).

These results were found to be consistent throughout the screen of $>20$ different populations of freshly isolated HSCs and therefore demonstrate a reliable and flexible experimental approach. This system was therefore explored to study the biochemical implication of cathepsin $S$ in the molecular mechanisms associated with HSC differentiation into dendritic cells along the immunogenic or immunosuppressive pathway (Martino et al., submitted manuscript).

Together, our findings point to DOTAP as a chemical suitable for siRNA delivery into HSCs, in light of a transfection efficiency $(\sim 60 \%)$ comparable to the transduction rate recently achieved in the same human cells through lentiviral vectors [17], and the excellent tolerance to repeated exposures. The ability to dramatically enhance antisense oligodeoxyribonucleotide uptake had, in previous studies, been taken as evidence for DOTAP's biocompatibility with HSCs [28]. However, absence of any disruption during HSC differentiation further suggests that the DOTAP protocol herein described may be successfully extended to other HSCrelated systems, including models aimed at addressing existing controversial perspectives on HSC transdifferentiation and overall plasticity $[29,30]$.

It is possible that other chemical agents, not included in our screen, may exhibit a performance comparable, if not better, to that produced by DOTAP. In this regard, the cationic lipophosphoramide KLN-5 was shown to be a nontoxic, highly efficient vehicle for transgene delivery into HSCs [31]; however, this chemical has not yet been tested in repeated siRNA transfection procedures and, to our knowledge, is not widely available through vendors. It must be noted that while a large number of lipid formulations could be designed to introduce nucleic acids in cells, a complex combination of chemical-physical and biological factors may severely limit the selection of chemicals capable of transfecting synthetic siRNAs with high transfection scores in HSCs. Indeed, cationic liposomes such as DOTAP, Lipofectamine 2000 and Lipofectamine LTX 

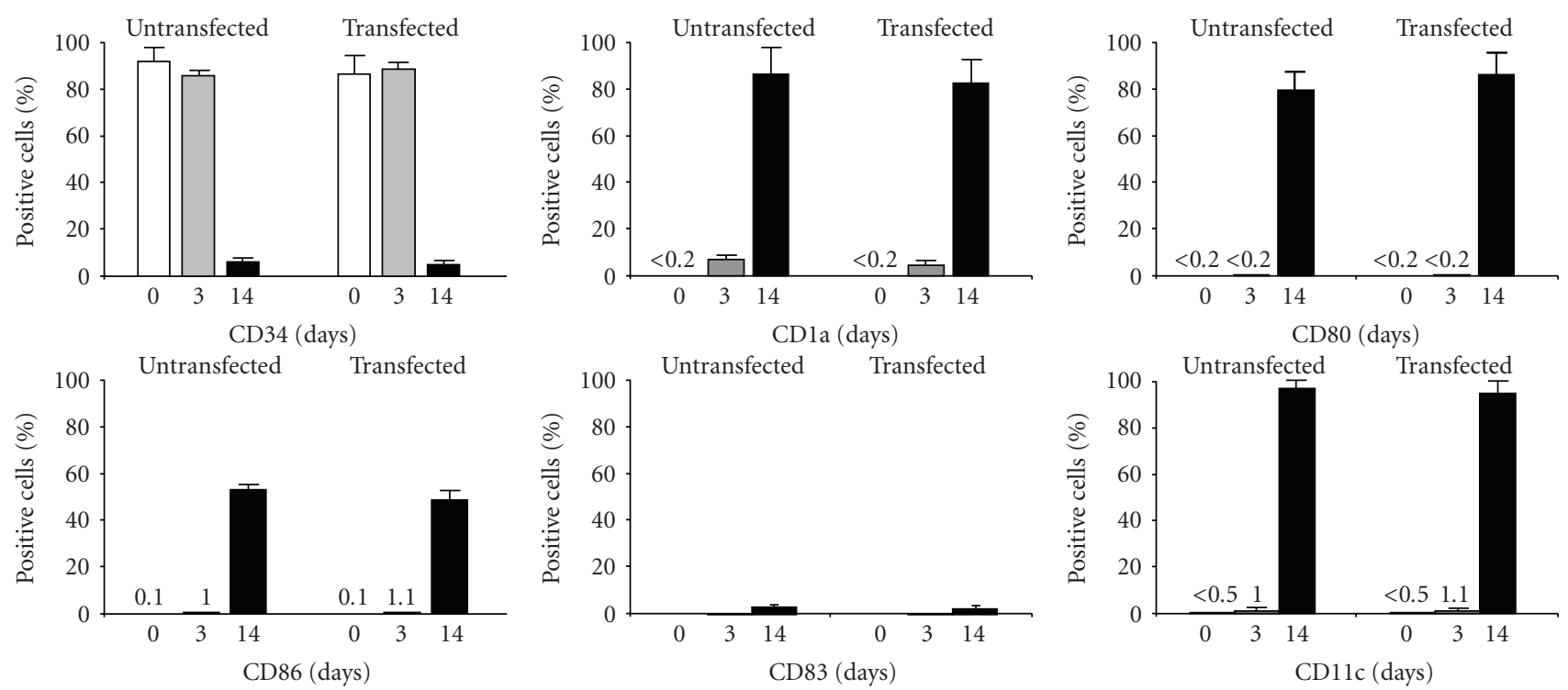

FIGURE 3: Expression of phenotypic markers throughout HSC differentiation into immunogenic dendritic cells in presence of multiple, DOTAPmediated transfection rounds. HSCs were subjected to differentiation agents, and then immediately split into two parallel cultures, one of which was maintained in the absence of manipulations, while the other was subjected to DOTAP-based, scrambled siRNA transfection rounds. Phenotypic antigens typically modulated during HSC differentiation into immunogenic dendritic cells were analyzed in HSCs at day 0 (white bars), day 3 (grey bars), and day 14 (black bars). In all instances, analyses were performed through a FACScan flow cytometer using monoclonal antibodies labeled with fluorescein isothiocyanate or phycoerythrin. Data shown are representative of triplicate determinations.

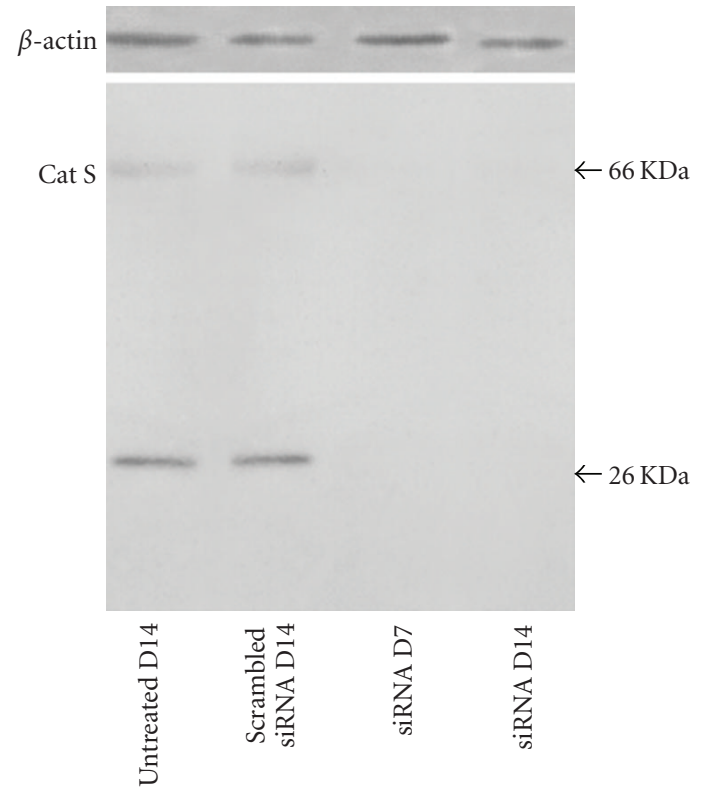

(a)

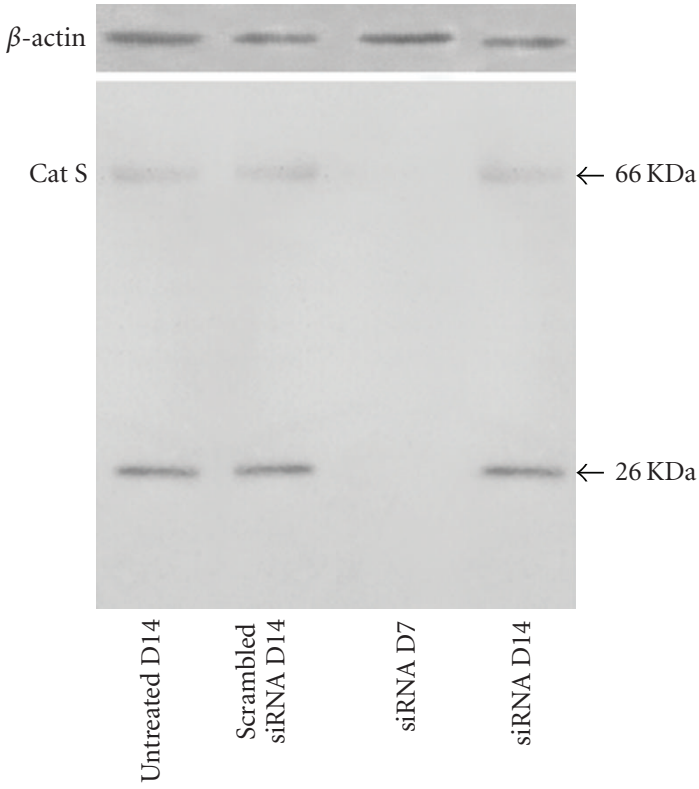

(b)

FIGURE 4: Silencing of cathepsin S during HSC differentiation. (a) Levels of cathepsin S were assessed by Western blotting in dendritic cells generated from the differentiation in vitroof HSCs. During the 14-day differentiation process, cells were subjected to DOTAP-based transfection rounds on day 0, 3, and 9 using a CTSS-targeting siRNA as described in the Materials and Methods. Untreated and scrambled siRNA-transfected cells were employed for control purposes. Levels of precursor ( $66 \mathrm{KDa}$ ) and mature (26 KDa) forms of cathepsin $\mathrm{S}$ at different time points (i.e., days 7 and 14) are indicated. $\beta$-actin was used to normalize the signals. (b) Shown is the same analysis as in panel A, except for the number of transfections, that were performed on day 0 and 3 only. Graphs are representative of experiments consistently reproduced using at least 20 different populations of HSCs. 
display a positively-charged headgroup that engages electrostatic interactions with the sugar-phosphate backbone, a spacer that may or may not be designed to facilitate such interactions, and 1-3 hydrocarbon chains that may vary in length, extent of saturation and distribution of cis and trans configurations [32]. Thus, cationic liposomes can be subject to a high degree of structural permutations, likely correlated with a capacity to transfer nucleic acids in specific cell types. Despite structural similarities and a shared clathrinmediated endocytosis process for DNA delivery [33, 34], these reagents exhibited a very wide performance range when tested in the HSC system. Marked differences in vector efficiencies may also arise from different mechanisms of internalization linked to subsequent, intracellular trafficking routes associated with macromolecular fates [33].

\section{Conclusions}

Our findings clearly point to DOTAP as an ideal vehicle for in vitro studies that require gene expression modulation in HSCs. It will, therefore, be intriguing to investigate whether this reagent can also be adapted to in vivo applications, for example, through combination with low-molecularweight polyethylenimines. These may indeed synergize with liposomes for DNA uptake [35] and have already been proven to be effective in preclinical models [36, 37]. This approach, if successful, would obviate the safety concerns associated with viral-mediated insertional mutagenesis.

\section{Acknowledgments}

The authors would like to thank Anna C. Berardi from IRCCS-Bambino Gesù Roma, Italy, for useful discussions and comments on the manuscript. This work was supported by Grants from MIUR (FIRB- Idea Progettuale n.RBIP06FH7J_002), Fondazione Cassa Risparmio di Perugia (Project 2007.0149.02), Ministero della Salute (Project n, RF-UMB-2006-339457), and Consorzio INNB to A.O.

\section{References}

[1] S. E. Martin and N. J. Caplen, "Applications of RNA interference in mammalian systems," Annual Review of Genomics and Human Genetics, vol. 8, pp. 81-108, 2007.

[2] D. E. Root, N. Hacohen, W. C. Hahn, E. S. Lander, and D. M. Sabatini, "Genome-scale loss-of-function screening with a lentiviral RNAi library," Nature Methods, vol. 3, no. 9, pp. 715719, 2006.

[3] M. Boutros and J. Ahringer, "The art and design of genetic screens: RNA interference," Nature Reviews Genetics, vol. 9, no. 7, pp. 554-566, 2008.

[4] D. Bumcrot, M. Manoharan, V. Koteliansky, and D. W. Y. Sah, "RNAi therapeutics: a potential new class of pharmaceutical drugs," Nature Chemical Biology, vol. 2, no. 12, pp. 711-719, 2006.

[5] A. Orlacchio, G. Bernardi, A. Orlacchio, and S. Martino, "RNA interference as a tool for Alzheimer's disease therapy," MiniReviews in Medicinal Chemistry, vol. 7, no. 11, pp. 1166-1176, 2007.
[6] A. Orlacchio, G. Bernardi, A. Orlacchio, and S. Martino, "Patented therapeutic RNAi strategies for neurodegenerative diseases of the CNS," Expert Opinion on Therapeutic Patents, vol. 18, no. 10, pp. 1161-1174, 2008.

[7] D. H. Kim and J. J. Rossi, "Strategies for silencing human disease using RNA interference," Nature Reviews Genetics, vol. 8, no. 3, pp. 173-184, 2007.

[8] S. M. Elbashir, J. Harborth, W. Lendeckel, A. Yalcin, K. Weber, and T. Tuschl, "Duplexes of 21-nucleotide RNAs mediate RNA interference in cultured mammalian cells," Nature, vol. 411, no. 6836, pp. 494-498, 2001.

[9] D.-H. Kim, M. A. Behlke, S. D. Rose, M.-S. Chang, S. Choi, and J. J. Rossi, "Synthetic dsRNA Dicer substrates enhance RNAi potency and efficacy," Nature Biotechnology, vol. 23, no. 2, pp. 222-226, 2005.

[10] J. Moffat, J. H. Reiling, and D. M. Sabatini, "Off-target effects associated with long dsRNAs in Drosophila RNAi screens," Trends in Pharmacological Sciences, vol. 28, no. 4, pp. 149-151, 2007.

[11] S. A. Stewart, D. M. Dykxhoorn, D. Palliser, et al., "Lentivirusdelivered stable gene silencing by RNAi in primary cells," RNA, vol. 9, no. 4, pp. 493-501, 2003.

[12] M. Li and J. J. Rossi, "Lentiviral vector delivery of siRNA and shRNA encoding genes into cultured and primary hematopoietic cells," Methods in Molecular Biology, vol. 433, pp. 287-299, 2008.

[13] O. ter Brake, K. 't Hooft, Y. P. Liu, M. Centlivre, K. J. von Eije, and B. Berkhout, "Lentiviral vector design for multiple shRNA expression and durable HIV-1 inhibition," Molecular Therapy, vol. 16, no. 3, pp. 557-564, 2008.

[14] R. S. Pillai, S. N. Bhattacharyya, and W. Filipowicz, "Repression of protein synthesis by miRNAs: how many mechanisms?" Trends in Cell Biology, vol. 17, no. 3, pp. 118-126, 2007.

[15] S. Vasudevan, Y. Tong, and J. A. Steitz, "Switching from repression to activation: microRNAs can up-regulate translation," Science, vol. 318, no. 5858, pp. 1931-1934, 2007.

[16] D. H. Kim, P. Sætrom, O. Snøve Jr., and J. J. Rossi, "MicroRNAdirected transcriptional gene silencing in mammalian cells," Proceedings of the National Academy of Sciences of the United States of America, vol. 105, no. 42, pp. 16230-16235, 2008.

[17] F. Di Nunzio, B. Piovani, F.-L. Cosset, F. Mavilio, and A. Stornaiuolo, "Transduction of human hematopoietic stem cells by lentiviral vectors pseudotyped with the RD114-TR chimeric envelope glycoprotein," Human Gene Therapy, vol. 18, no. 9, pp. 811-820, 2007.

[18] L. H. Li, P. McCarthy, and S. W. Hui, "High-efficiency electrotransfection of human primary hematopoietic stem cells," The FASEB Journal, vol. 15, no. 3, pp. 586-588, 2001.

[19] J. M. Wiehe, P. Ponsaerts, M. T. Rojewski, et al., "mRNAmediated gene delivery into human progenitor cells promotes highly efficient protein expression," Journal of Cellular and Molecular Medicine, vol. 11, no. 3, pp. 521-530, 2007.

[20] V. F. I. Van Tendeloo, H.-W. Snoeck, F. Lardon, et al., "Nonviral transfection of distinct types of human dendritic cells: highefficiency gene transfer by electroporation into hematopoietic progenitor- but not monocyte-derived dendritic cells," Gene Therapy, vol. 5, no. 5, pp. 700-707, 1998.

[21] G. Ferlazzo, J. Klein, X. Paliard, W.-Z. Wei, and A. Galy, "Dendritic cells generated from CD $34^{+}$progenitor cells with flt3 ligand, c-Kit ligand, GM-CSF, IL-4, and TNF- $\alpha$ are functional antigen-presenting cells resembling mature monocyte-derived dendritic cells," Journal of Immunotherapy, vol. 23, no. 1, pp. $48-58,2000$. 
[22] R. Tiribuzi, S. Martino, E. Ciraci, et al., "Non-redundant roles of cathepsins $\mathrm{L}, \mathrm{B}$ and $\mathrm{S}$ in $\mathrm{CDla}^{+}$dendritic cells knocked-down for cathepsin S by RNA interference," Minerva Biotecnologica, vol. 20, no. 2, pp. 59-67, 2008.

[23] S. Della Bella, S. Nicola, I. Timofeeva, M. L. Villa, A. Santoro, and A. C. Berardi, "Are interleukin-16 and thrombopoietin new tools for the in vitro generation of dendritic cells?" Blood, vol. 104, no. 13, pp. 4020-4028, 2004.

[24] S. Martino, C. Emiliani, A. Orlacchio, R. Hosseini, and J. L. Stirling, " $\beta-N$-acetylhexosaminidases $\mathrm{A}$ and $\mathrm{S}$ have similar sub-cellular distributions in HL-60 cells," Biochimica et Biophysica Acta, vol. 1243, no. 3, pp. 489-495, 1995.

[25] K. Honey and A. Y. Rudensky, "Lysosomal cysteine proteases regulate antigen presentation," Nature Reviews Immunology, vol. 3, no. 6, pp. 472-482, 2003.

[26] L. Delamarre, M. Pack, H. Chang, I. Mellman, and E. S. Trombetta, "Differential lysosomal proteolysis in antigenpresenting cells determines antigen fate," Science, vol. 307, no. 5715, pp. 1630-1634, 2005.

[27] D. A. Scudiero, R. H. Shoemaker, K. D. Paull, et al., "Evaluation of a soluble tetrazolium/formazan assay for cell growth and drug sensitivity in culture using human and other tumor cell lines," Cancer Research, vol. 48, no. 17, pp. 4827-4833, 1988.

[28] R. Kronenwett, U. Steidl, M. Kirsch, G. Sczakiel, and R. Haas, "Oligodeoxyribonucleotide uptake in primary human hematopoietic cells is enhanced by cationic lipids and depends on the hematopoietic cell subset," Blood, vol. 91, no. 3, pp. 852-862, 1998.

[29] D. Orlic, J. Kajstura, S. Chimenti, et al., "Bone marrow cells regenerate infarcted myocardium," Nature, vol. 410, no. 6829, pp. 701-705, 2001.

[30] C. E. Murry, M. H. Soonpaa, H. Reinecke, et al., "Haematopoietic stem cells do not transdifferentiate into cardiac myocytes in myocardial infarcts," Nature, vol. 428, no. 6983, pp. 664$668,2004$.

[31] T. Montier, P. Delépine, K. Le Ny, et al., "KLN-5: a safe monocationic lipophosphoramide to transfect efficiently haematopoietic cell lines and human CD $34^{+}$cells," Biochimica et Biophysica Acta, vol. 1665, no. 1-2, pp. 118-133, 2004.

[32] X. Gao and L. Huang, "Cationic liposome-mediated gene transfer," Gene Therapy, vol. 2, no. 10, pp. 710-722, 1995.

[33] A. W. Wong, S. J. Scales, and D. E. Reilly, "DNA internalized via caveolae requires microtubule-dependent, Rab7-independent transport to the late endocytic pathway for delivery to the nucleus," The Journal of Biological Chemistry, vol. 282, no. 31, pp. 22953-22963, 2007.

[34] J. Rejman, A. Bragonzi, and M. Conese, "Role of clathrin- and caveolae-mediated endocytosis in gene transfer mediated by lipo- and polyplexes," Molecular Therapy, vol. 12, no. 3, pp. 468-474, 2005.

[35] P. Lampela, P. Soininen, A. Urtti, P. T. Männistö, and A. Raasmaja, "Synergism in gene delivery by small PEIs and three different nonviral vectors," International Journal of Pharmaceutics, vol. 270, no. 1-2, pp. 175-184, 2004.

[36] A. Bragonzi, G. Dina, A. Villa, et al., "Biodistribution and transgene expression with nonviral cationic vector/DNA complexes in the lungs," Gene Therapy, vol. 7, no. 20, pp. 17531760, 2000.

[37] H.-W. Liao and K.-W. Yau, "In vivo gene delivery in the retina using polyethylenimine," BioTechniques, vol. 42, no. 3, pp. 285-288, 2007. 

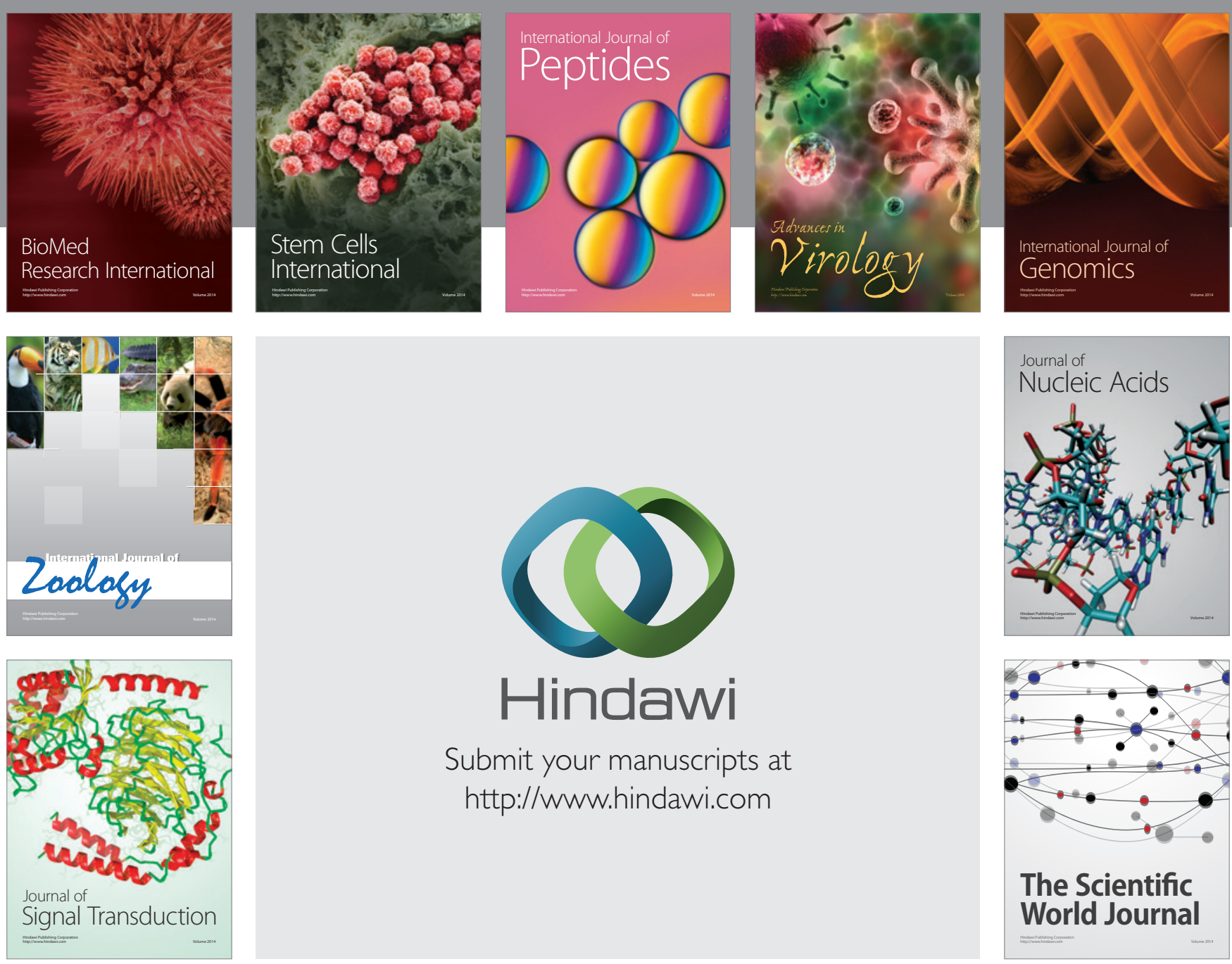

Submit your manuscripts at

http://www.hindawi.com
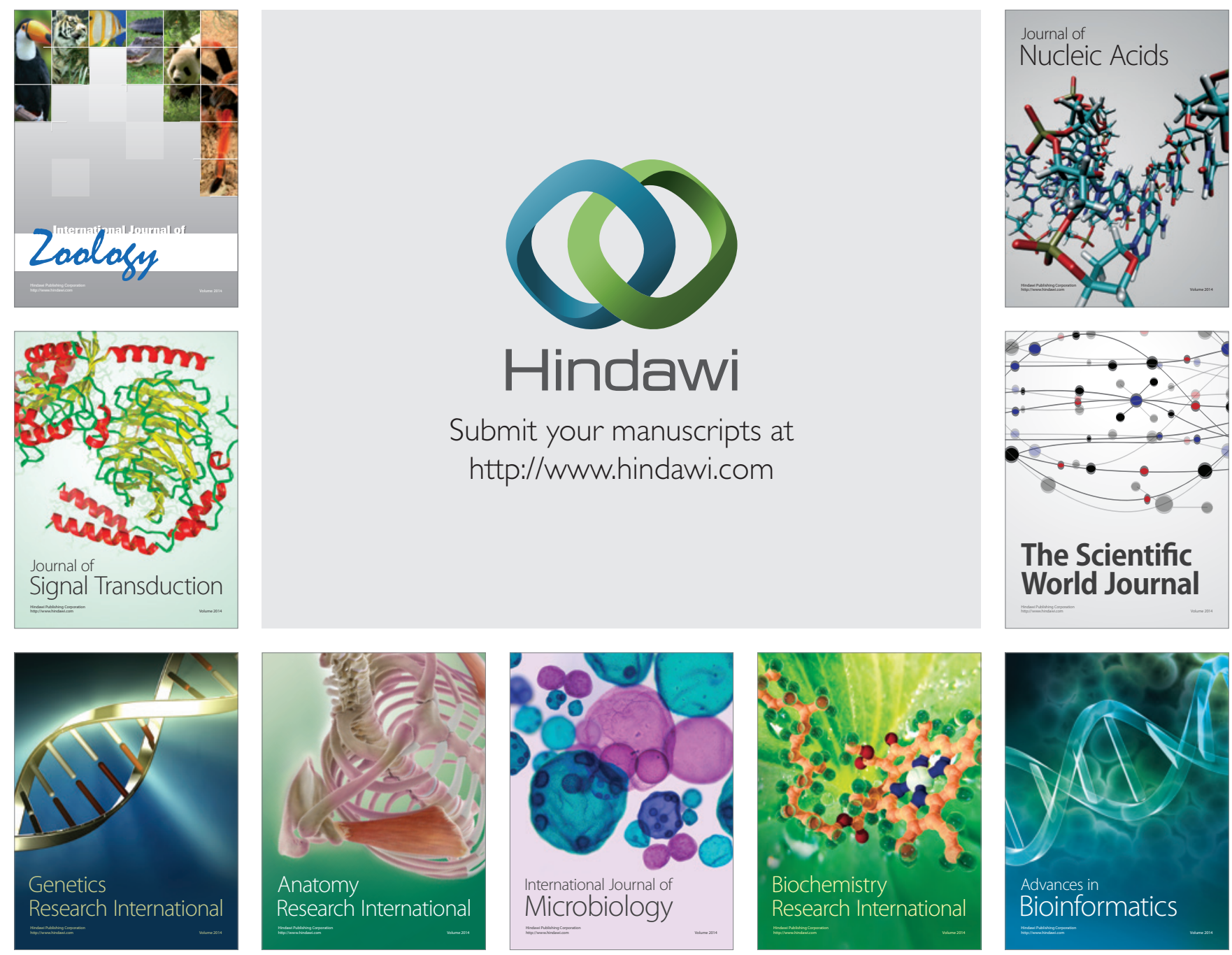

The Scientific World Journal
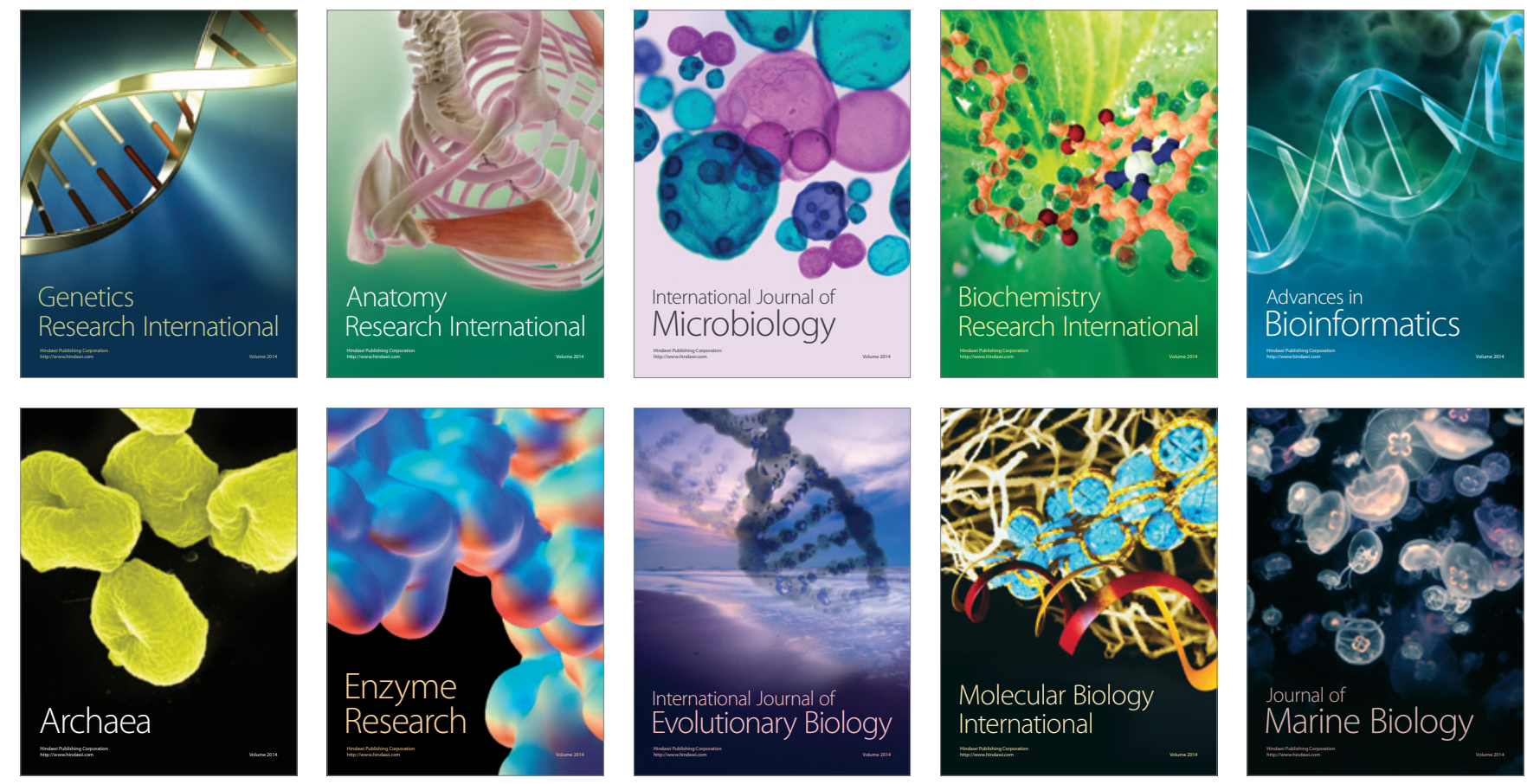